\title{
Deciphering the Role of Quantum Dots Size in the Ultrafast Charge Carrier Dynamics at the Perovskite-Quantum Dots Interface
}

P. Piatkowski, ${ }^{1,2}$ Sofia Masi, ${ }^{3}$ Pavel Galar, ${ }^{1 \#}$ Mario Gutierrez Tovar, ${ }^{1 \&}$ Thi Tuyen Ngo, ${ }^{3}$ Iván Mora Seró, ${ }^{3 *}$ Abderrazzak Douhal ${ }^{1 *}$

${ }^{1}$ Departamento de Química Física, Facultad de Ciencias Ambientales y Bioquímica, INAMOL, Universidad de Castilla-La Mancha, Avenida Carlos III, 45071 Toledo, Spain.

${ }^{2}$ Department of Chemistry, University of Warsaw, Zwirki I Wigury 101, Warsaw 02-089, Poland.

${ }^{3}$ Institute of Advanced Materials (INAM), Universitat Jaume I, Av. de VicentSosBaynat, S/N, 12006, Castelló de la Plana, Spain.

*Corresponding authors: (IMS) sero@uji.esand (AD) abderrazzak.douhal@uclm.es

\# Present address: Institute of Physics, Czech Academy of Sciences, 10/112, 16200 Prague 6, Czech Republic.

\& Present address: Multifunctional Materials \& Composites (MMC) Laboratory, Department of Engineering Science, University of Oxford, Parks Road, Oxford OX1, UK. 


\section{Abstract}

Understanding the behaviour of electrons and holes $(\mathrm{e}, \mathrm{h})$ diffusion and transfer at the interfaces of photoexcited hybrid materials at different densities of photoexcited charge carriers is paramount to the development of efficient optoelectronic devices. Nanocomposites formed by methylammonium lead iodide perovskite $\left(\mathrm{MAPbI}_{3}\right)$ and semiconductor colloidal quantum dots (QDs)are among these hybrid materials under intensive studies. However, the reciprocal influence of the components in the composite material on the temporal evolution of the photoinduced charge carriers is still poorly explored. This study explores the ultrafast temporal behaviour of the photoexcited charge carriers in $\mathrm{MAPbI}_{3} / \mathrm{PbS}$ QDs films, letting a special attention to the role of the $\mathrm{PbS} \mathrm{QD}$ size. Armed with fs-time-resolved UV-VIS transient absorption and terahertz techniques, we unravel the effect of different sizes of $\mathrm{PbS}$ QDs, embedded in perovskite (PS) host matrix, on the processes of e and h diffusion, transfer to the QDs phase and recombination. While the decays are dominated by e and $\mathrm{h}$ transition from PS to QDs, the increase in the size of QDs results in an acceleration of the charge carriers transition processes represented by the total transition rate constants of electrons $\left(\mathrm{k}_{\mathrm{e}}\right)$ and holes $\left(\mathrm{k}_{\mathrm{h}}\right)$. The total $\mathrm{k}_{\mathrm{e}}$ and $\mathrm{k}_{\mathrm{h}}$ values change form 0.1 and $1\left(10^{9} \mathrm{~s}^{-1}\right)$ to 4.5 and $22\left(10^{9} \mathrm{~s}^{-1}\right)$, respectively. We extract the rate constants of their diffusions $\left(\mathrm{k}_{\text {ediff }}=2.2 \times 10^{10} \mathrm{~s}^{-1}\right.$ and $\mathrm{k}_{\text {hdiff }}=$ $\left.1.1 \times 10^{10} \mathrm{~s}^{-1}\right)$ and transfers to the interfaces $\left(\mathrm{k}_{\mathrm{et}}=0.1\right.$ to $1.6 \times 10^{10} \mathrm{~s}^{-1}$ and $\mathrm{k}_{\mathrm{ht}}=0.1$ to $0.8 \times$ $\left.10^{10} \mathrm{~s}^{-1}\right)$. Furthermore, the analysis of spectral behavior of PS and PS/QDs upon pumping with different fs-laser fluences indicate the presence and photoformation of excitonic states. The acceleration of such processes decreases the contribution of undesirable charge carriers trapping and non-radiative recombination within PS.

Keywords: Perovskite; quantum dots; hybrid nanostructures; ultrafast electron and hole dynamics, interfaces.

\section{Introduction}


Combining smart materials of different nature, like halide perovskites (PS) and semiconductor colloidal quantum dots(QDs), could provide potential synergistic interaction allowing to overcome limitations of each component, and thus, enhancing the performance of the related devices. ${ }^{[1-2]}$ PS/QDs hybrids constitute one interesting example with important optoelectronic applications that have been successfully used in the fabrication of IR LEDs, ${ }^{[3-6]}$ solar cells ${ }^{[7-11]}$ and photodetectors. ${ }^{[12-13]}$ Halide perovskite can passivate the surface of QDs enhancing the performance of colloidal QD solar cells ${ }^{[14-15]}$ while PbS QDs can act as seeds in the growth of PS enhancing the properties of the perovskite layer ${ }^{[9,16-19]}$

Potential applications for the development of advanced optoelectronic device configurations of this kind of hybrid materials are demonstrated using methylammonium lead triiodide $\left(\mathrm{MAPbI}_{3}\right)$ perovskite matrix containing PbS/CdS core/shell QDs. ${ }^{[4]}$ However, due to the complex nature and structure of these hybrids interface, the improvement of their electrooptical devices needs a deeper understanding of the involved charge carrier dynamics at their interfaces, which shapes and quantifies their performance. To this end, ultrafast spectroscopic techniques are powerful tools to unveil the interaction between different materials. Recently, we have used UV-VIS transient absorption (TA) and terahertz (THz) spectroscopy to characterize the interaction of $\mathrm{MAPbI}_{3}$ with embedded $\mathrm{PbS} / \mathrm{CdS}$ colloidal QDs considering different densities of QDs. ${ }^{[19]}$ The study showed the important role of the QDs electronic states, especially trap states, on the deactivation dynamics of the charge carriers photoexcited in perovskite matrix.

However, important aspects of the QD versatility as the effect in the material composite of the QD size remain unexplored. One of the interesting properties of the colloidal QDs is the easy tuning of the band gap by a control of the QD size ${ }^{[20-21]}$ It was reported that the change in the size of $\mathrm{PbS}$ QDs in PS/QDs nanocomposite from 2.3 to $3.0 \mathrm{~nm}$ results in strong shift of position of electroluminescence, which demonstrates the tunability of optical 
properties in such systems. ${ }^{[4]}$ Fine tuning of the band gap in QDs is important to achieve efficient transfer of both electron and hole from PS to QDs followed by light emission in IRLED. The utilization of such properties might result in tunable infrared LED devices. The band gap is a key parameter in order to understand the interaction between different materials as it affects the alignment of the energy bands, and consequently the charge transfer properties. Moreover, the decryption of the influence of the interface between PS and $\mathrm{PbS}$ that is a source of electron, hole and excitonic trap states on the overall properties of the nanocomposite, allows detailed studies of the composite materials using different densities of the photoexcited charge carriers. ${ }^{[16]}$ However, up to date, there is no systematic study of the interaction of PS with QDs of different size.

Herein, we elucidate the influence of the PbS QDs size (2.9, 3.2, 4.5 and $5.3 \mathrm{~nm}$ ), when the QDs are embedded in PS matrix, and probe the ultrafast dynamics of photoinduced electrons and holes, when migrating and transferring to the interface between both materials. We used fs-time-resolved Vis-NIR transient absorption (TA), terahertz (THz) and nanosecond flash photolysis spectroscopies. The present $\mathrm{PbS}$ QDs are different from the ones using core/shell PbS/CdS nanocrystals in our previous work. ${ }^{[16]}$ We have found that photogenerated electrons and holes in PS are rapidly transferred to PbS QDs independently on the size of nanostructures. Remarkably, the dynamics of these processes is strongly dependent on QD size. By the use of a kinetic model we extract the total rate constants of electrons $\left(\mathrm{k}_{\mathrm{e}}\right)$ and hole $\left(\mathrm{k}_{\mathrm{h}}\right)$. We unravel that both $\mathrm{k}_{\mathrm{h}}$ and $\mathrm{k}_{\mathrm{e}}$ increase from 0.1 to $4.5 \times\left(10^{9} \mathrm{~s}^{-1}\right)$ and from 0.1 to 2.2 $\times\left(10^{10} \mathrm{~s}^{-1}\right)$, respectively, upon increasing the size of QDs. This reflects a more efficient charge carriers transfer form PS to nanostructures. The results indicate that electron is transferred from PS to PbS QDs faster than the hole. In contrast to previous report on core/shell $\mathrm{PbS} / \mathrm{CdS}$ nanocrystals embedded in PS. ${ }^{[16]}$ Based on total rate constants along with THz results we extracted, the values of electrons and holes diffusion $\left(\mathrm{k}_{\text {ediff }}=2.2 \times 10^{10} \mathrm{~s}^{-1}\right.$ and $\mathrm{k}_{\text {hdiff }}=1.1 \times$ 
$\left.10^{10} \mathrm{~s}^{-1}\right)$ and transfer $\left(\mathrm{k}_{\text {et }}=0.1\right.$ to $1.6 \times\left(10^{10} \mathrm{~s}^{-1}\right)$ and $\mathrm{k}_{\mathrm{ht}}=0.1$ to $0.8 \times\left(10^{10} \mathrm{~s}^{-1}\right)$ rate constants within the systems containing different sizes of QDs. The acceleration of charge carriers transfer from PS to QD decreases the probability of non-radiative deactivation within PS and thus for efficient LED devices based on PS/QDs. The spectral analysis of the TA signal at different pump fluences shows the presence of excitons in all studied samples as well as the photoformation of new excitonic states upon increasing the fluence of the absorbed photons in PS and PS/QDs samples. Furthermore, the larger contribution of excitonic states in PS/QDs films as related to neat PS is of relevance to the development of photovoltaic devices.

\section{Result and discussion}

\subsection{Transient absorption studies}

We first present and discuss the results of TA studies of neat PS and PS films containing PbS QDs. The samples were fs-pumped at $600 \mathrm{~nm}$ using a fluence of the absorbed photons of $8.2 \times 10^{12} \mathrm{ph} / \mathrm{cm}^{2}$ and probed from $620 \mathrm{~nm}$ to $780 \mathrm{~nm}$. Figure $\mathrm{S} 1$ shows representative TA spectra of neat PS and PS/QDs films gated at 1 ps pump-probe delay time. The $\mathrm{MAPbI}_{3}$ TA spectrum is composed of a positive signal ranging from $640 \mathrm{~nm}$ to $720 \mathrm{~nm}$, reflecting the absorption of photoexcited electrons and holes (PA), and a photobleaching band (PB) with minimum intensity at $760 \mathrm{~nm} \cdot{ }^{[22-24]}$ Interestingly, the presence of $\mathrm{PbS}$ nanocrystals does not affect the shape and the position of both TA bands of PS/QDs film suggesting that the photoresponse of the system, during the initial charge generation and cooling time period, originates only from $\mathrm{PS}$ host matrix. $\mathrm{PbS}$ and $\mathrm{MAPbI}_{3}$ possess the same six-coordinated $\mathrm{Pb}$ atom and they have similar $\mathrm{Pb}-\mathrm{Pb}$ distances $(6.26 \AA$ and $5.95 \AA$ in case ofMAPbI 3 and $\mathrm{PbS}$, respectively), that gives good structural conditions for minimizing the QDs/host matrix interfacial strain, and explain the results presented in Figure $1 .^{[3,7,25]}$ In addition to similar shape, a further monitoring of the TA signals in PS and PS/QDs samples shows significant 
differences, which are discussed below in the analysis of their TA decays at specific wavelength.

Figures 1a-e show the temporal evolution of the TA spectra upon excitation at $600 \mathrm{~nm}$, while Figure S2 presents single TA decays observed at $760 \mathrm{~nm}$ (maximum of PB band). Clearly, the presence of QDs embedded in PS film results in faster deactivation of the photoexcited charge carriers in PS host due to diffusion and subsequent transfer of electrons and holes form PS to QDs. ${ }^{[16]}$ In addition to that, the decay time becomes shorter upon increasing the size of the $\mathrm{PbS}$ nanostructures.

Previous TA experiments on PS/QDs, with QDs size of $3 \mathrm{~nm}$, clearly showed that the TA of the nanocomposite film is related only to the electrons and holes present within the PS host, ${ }^{[16]}$ and this response was influenced by the concentration of QDs. In the present work, the preparation conditions of the materials were set to obtain similar concentrations of the $\mathrm{PbS}$ QDs of different size in different PS/QDs samples, and thus, we target the effect of the QD size in the photogenerated dynamics.

Increasing the QD size can result in higher interfacial defects which act as trap carrier centers. ${ }^{[26]}$ In addition to that, there is also a shape evolution from octahedron for small size QDs (1- $3 \mathrm{~nm}$ ) having surfaces of (111) to cuboctahedron shape for QDs with size larger than $\sim 4 \mathrm{~nm}$ of surfaces with both (111) and (100) orientation. ${ }^{[27]}$ Hence increasing the size of embedded PbS QDs might induce a higher concentration of interfacial trap states at PS/QDs interfaces. On the other hand, as Figure 1f shows increasing the QDs size leads to a change of valence (VB) and conduction $(\mathrm{CB})$ bands of $\mathrm{PbS} \mathrm{QDs}$, due to a reduction of quantum confinement. Thus, the occurrence of charge carrier's migration from PS results in faster TA signal decays.

For all the used nanocomposite systems, the VB in PS is located below in the one of QDs (Figure 1f).Thus, the holes from PS VB are most probably transferred to the VB in the 
nanostructure (Scheme 1). ${ }^{[4,28-30]}$ In the case of photogenerated electrons, the nature of processes of the electron transition differs for the QDs of different sizes. For the systems which contain QDs of 2.9 and $3.2 \mathrm{~nm}$, the $\mathrm{CB}$ of PS is located lower than that in $\mathrm{PbS}$ nanostructure, and thus the photoexcited electrons in the PS cannot move into the CB of the QDs (Scheme 1a). However, our previous report suggests that in such situation, the electrons are efficiently transferred to interfacial electronic states formed between PS and PbS QDs. ${ }^{[16]}$ In the case of nanocomposites which contain bigger QDs $(4.5 \mathrm{~nm}, 5.3 \mathrm{~nm})$, the CB of PS is located slightly below that of $\mathrm{PbS}$, allowing electron transition into the $\mathrm{CB}$ of QDs (Scheme 1b).

From Figures1 and S2, it is evident that the TA signals of different nanocomposites (QDs of $3.2-5.3 \mathrm{~nm}$ diameter) decay to small values in $1.5 \mathrm{~ns}$. While the effective masses of electrons and holes in PS are comparable, the photoresponse in TA experiment should be caused by both charge carriers within the $1.5 \mathrm{~ns}$ time window. ${ }^{[24,31-32]}$ Hence, the observed dynamics suggest that both electrons and holes are transferred from the PS host independently on the size of the QDs. Considering the TA decay of neat PS depicted in Figure S2, where small changes are observed within 1.5 ns time range, the contribution of charge carrier recombination within the PS host matrix on observed dynamics in PS/QDs should be marginal.

\subsection{Rate constants of electron and holes dynamics}

To quantify the dynamics related to the photoevents observed in the nanocomposites, we have used the model described in our previous work, ${ }^{[16]}$ where the TA dynamics are dominated by free charge carriers that are formed directly after photoexcitation of PS. ${ }^{[33-35]}$ Accordingly, the interrogated sample can be effectively separated into two regions where photoexcited charges $(i)$ are affected by the presence of QDs (region $\mathrm{A}_{1}$ ) and (ii) behave like in neat PS (region $A_{2}$ ). We assume that the recombination processes within PS host are 
characterized by rate constants similar to those for neat $\mathrm{MAPbI}_{3}$. Hence, we have used below equation to calculate second ( $\left.k_{\text {bimol }}\right)$ and third order $\left(k_{\text {Auger }}\right)$ rate constants in neat PS and the resulted values were used in the model describing nanocomposite materials: ${ }^{[22,36-37]}$

$\frac{\mathrm{d} n}{\mathrm{dt}}=-k_{\mathrm{mono}} n-k_{\mathrm{bimol}} n^{2}-k_{\text {Auger }} n^{3}$,

where $n$ is the excited charge carrier's concentration. The first order rate constant $\left(k_{\text {mono }}\right)$ of neat PS and PS/QDs samples were extracted from multiexponential fits to the flash photolysis results (Figure S3 and Table S2). The fit of the experimental data of PS gives the following values: $k_{\text {mono }}=1.59 \times 10^{7} \mathrm{~s}^{-1}, k_{\text {bimol }}=1.28 \times 10^{-11} \mathrm{~s}^{-2}$ and $k_{\text {Auger }}=2.75 \times 10^{-29} \mathrm{~s}^{-3}$. The obtained rate constants are in good agreement with previous reports. ${ }^{[1,22,34,38]}$

Obviously, in the presence of QDs new processes appear and the photoexcited charge carriers are deactivated much faster. ${ }^{[16]}$ As we mentioned, the acceleration of the decay is due to efficient and fast electrons and holes transfer from perovskite to QDs. Furthermore, the presence of QDs favors the formation of interfacial excitonic levels, and thus the contribution of excitons to the decay of TA signal is taken into account. The equations of the kinetic model for recombination processes in PS/QDs system are given below: ${ }^{[16]}$

$$
\begin{aligned}
& \frac{\mathrm{d} n_{\mathrm{e}}}{\mathrm{d} t}=G_{\mathrm{e}}-n_{\mathrm{e}} k_{\text {mono }}-n_{\mathrm{e}} n_{\mathrm{h}} k_{\mathrm{bimol}}-n_{\mathrm{e}}^{2} n_{\mathrm{h}} k_{\text {Auger }}-n_{\mathrm{e}} k_{\mathrm{e}}, \\
& \frac{\mathrm{d} n_{\mathrm{h}}}{\mathrm{d} t}=G_{\mathrm{e}}-n_{\mathrm{h}} k_{\text {mono }}-n_{\mathrm{e}} n_{\mathrm{h}} k_{\text {bimol }}-n_{\mathrm{h}}^{2} n_{\mathrm{e}} k_{\text {Auger }}-n_{\mathrm{h}} k_{\mathrm{h},} \\
& \frac{\mathrm{d} n_{\mathrm{ex}}}{\mathrm{d} t}=G_{\mathrm{x}}-n_{\mathrm{ex}} k_{\mathrm{ex}}, \\
& \frac{\mathrm{d} n_{\text {interface }}}{\mathrm{d} t}=n_{\mathrm{e}} k_{\mathrm{e}}-n_{\text {interface }} n_{\mathrm{QD}} k_{\mathrm{QDIn}}, \\
& \frac{\mathrm{d} n_{\mathrm{QD}}}{\mathrm{d} t}=n_{\mathrm{h}} k_{\mathrm{h}}-n_{\text {interface }} n_{\mathrm{QD}} k_{\mathrm{QDIn}},
\end{aligned}
$$

where $n_{\mathrm{e}}, n_{\mathrm{h}}$ and $n_{\mathrm{x}}$ are the concentrations of electrons, holes in CB and VB of PS and excitons close to the PS/QDs interface. $G_{\mathrm{e}}, G_{\mathrm{h}}$ and $G_{\mathrm{ex}}$ describe the generation factors of these populations. The electron and hole transfer processes from PS to PbS QDs are described by the first order total rate constants $k_{\mathrm{e}}$ and $k_{\mathrm{h}}$, respectively. ${ }^{[16,39]}$ These rate constants are related 
to transfers to $\mathrm{CB} /$ interface and to $\mathrm{VB}$, respectively. In the case PS/QDs (2.9, $3.2 \mathrm{~nm})$, for energetic conditions the migration of electron from PS's CB to the one of QD is impossible, and the electrons are trapped in the interface between the two materials (Scheme 1a). ${ }^{[16]}$ However, if the transition of charge carriers between VBs and CBs of the two components in PS/QDs systems is favored, the occurrence of simultaneous processes of electrons and holes trapping cannot be excluded. The migration of charge carriers to internal electronic states of QDs dominates the TA signal. Furthermore, due to confined nature of excitons they are not effectively migrating to QDs but recombine within PS which is described by $k_{\text {ex. }}$ Finally, in equations (2d) and (2e), $n_{\text {interface }}$ refers to the concentration of electrons transferred to the interface or to the $\mathrm{CB}$ of QDs, while $n_{\mathrm{QDIn}}$ reflects the hole transition to $\mathrm{VB}$ of $\mathrm{PbS}$. The recombination rate constant of these carriers is given by $\mathrm{k}_{\mathrm{QDIn}}$. Because the TA decays were gated at $760 \mathrm{~nm}$ result only from processes in PS, the last two equations (2d and 2e) are not considered in the calculations.

The recorded TA signals are given by the sum of the recombination processes in regions $\mathrm{A}_{1}$ and $\mathrm{A}_{2}$ :

$\Delta \mathrm{OD}=\mathrm{P}_{1}\left(\mathrm{n}_{\mathrm{e}}(\mathrm{t})+\mathrm{n}_{\mathrm{h}}(\mathrm{t})+\mathrm{n}_{\mathrm{ex}}(\mathrm{t})\right)+\mathrm{P}_{2}(\mathrm{n}(\mathrm{t}))$

where $P_{1}$ and $P_{2}$ are fractions of populations within areas affected $\left(\mathrm{A}_{1}\right)$ and non-affected $\left(\mathrm{A}_{2}\right)$ by the presence of QDs and $\mathrm{n}(\mathrm{t})$ is the charge carriers' concentrations in the area not affected by QDs.

Table 1 gives the extracted values of the rate constants for electrons, holes and excitons as well as their relative amplitudes in the TA signals of the studied composite materials having QDs of different sizes. First, the contribution of excitons population $\left(\mathrm{n}_{\mathrm{ex}}\right)$ to the total signal is negligible in all the decays. This reflects faster and more efficient transition of the charge carriers diffusing towards the surface of the nanostructures. As a result, the contribution of charge carriers $\left(\mathrm{P}_{1}\right)$ recombining in the area affected by the presence of 
$\operatorname{PbS}\left(\mathrm{A}_{1}\right)$ increases with the size of QDs. Remark that because of the mean distance between the QDs is similar in all the samples ( $25 \mathrm{~nm}$, see the supporting information for more details on the estimation), only the size of QDs is relevant to the observed behavior.

The electrons and holes in PS matrix are, in fact, generated at different distances from QDs, and thus they must diffuse towards the surface of the nanostructures before transfer to the PS/QDs interface or to VBs/CBs of QDs. Hence, the observed temporal evolution of TA signal in PS/QDs films represented by $k_{e}$ and $k_{h}$ in Equation 2 is due to both processes (Scheme 1). The total decay time of electrons $\left(\tau_{\mathrm{e}}=1 / \mathrm{k}_{\mathrm{e}}\right)$ and holes $\left(\tau_{\mathrm{h}}=1 / \mathrm{k}_{\mathrm{h}}\right)$ is a sum of diffusion and transfer times of electrons $\left(\tau_{\text {ediff }}=1 / \mathrm{k}_{\text {ediff }}, \tau_{\mathrm{et}}=1 / \mathrm{k}_{\mathrm{et}}\right)$ and holes $\left(\tau_{\text {hdiff }}=1 / \mathrm{k}_{\text {hdiff }}\right.$, $\left.\tau_{\mathrm{ht}}=1 / \mathrm{k}_{\mathrm{ht}}\right)$, and it is given by:

$\tau_{e}=\tau_{\text {ediff }}+\tau_{e t}$

$\tau_{h}=\tau_{\text {hiff }}+\tau_{h t}$

Our analysis clearly shows a significant increase in the values of the rate constants, $\mathrm{k}_{\mathrm{e}}$ and $\mathrm{k}_{\mathrm{h}}$, with the size of QDs (Table 1 and Figure 3). As, the mean distances between QDs in the studied nanocomposites are similar (21 - $25 \mathrm{~nm}$, Table S2), the observed differences between the values of $k_{e}, k_{h}$ are mostly due to increasing velocity of electron and hole transfer processes at the interface ( $\mathrm{k}_{\mathrm{et}}$ and $\left.\mathrm{k}_{\mathrm{ht}}\right)$. Only the values of $\mathrm{k}_{\mathrm{e}}\left(2.17 \times 10^{10} \mathrm{~s}^{-1}\right)$ are the same for QDs of 4.5 and $5.3 \mathrm{~nm}$. The increase in the size of the $\mathrm{PbS}$ nanocrystals results in a larger concentration and different energies of available electronic states, ${ }^{[40]}$ and therefore in an acceleration of deactivation processes related to the speed of charge carriers transition between PS and QDs. Thus, the observed changes in the values of $\mathrm{k}_{\mathrm{e}}$ and $\mathrm{k}_{\mathrm{h}}$ should depend on $\mathrm{k}_{\mathrm{et}}$ and $\mathrm{k}_{\mathrm{ht}}$ at the PS/QDs interface. The probability of charge carrier transition to QD also depends on the relative position of the energy states and their energy difference between PS and $\mathrm{PbS}$. For the used QDs, the VB is located above that in PS and the energy difference increases with the size of QDs (Scheme 1). As the driving force of charge carriers transition 
increases with difference in energy between the two partners, the time of hole transition into $\mathrm{PbS}$ becomes shorter (Figure 1 and S2). ${ }^{[41-43]}$ For $2.9 \mathrm{~nm}$ and $3.2 \mathrm{~nm}$ QDs, the CB is located above that of the PS, and as a result the electrons can be transferred only to the interfacial trap states (Scheme 1a). However, when the size of QDs is 4.5 and $5.3 \mathrm{~nm}$, the energy of the CB in the QDs is lower than that of PS CB, and thus the electrons are also transferred to the CB of the QD (Scheme 1b).

Because the equations related to the electrons and holes recombination in our model have the same structure, the model cannot give an answer to which value is related to which carrier (Equation 2a and 2b). However, as the VB in PS is located significantly below that in $\mathrm{PbS}$ nanocrystals, the holes can be efficiently transferred to QDs in all studied samples, see Figure 1f. ${ }^{[16]}$ Previously we have shown that when the CB in PS is below that of QDs, the electron could not migrate to this band. ${ }^{[16]}$ However, the decay of TA signal indicates that both charge carriers in the presence of $\mathrm{PbS} / \mathrm{CdS}$ are leaving the $\mathrm{CB} / \mathrm{VB}$ of $\mathrm{PS}$ in a faster way. We concluded that the electrons are trapped in the PS/QDs interface and the hole transition was expected to be faster than that of electron due to larger driving force. ${ }^{[16]}$ Nonetheless, the photobehaviour of the systems studied in the present report suggests that in all cases the electron transfer is much faster than the hole one. Direct comparison of the results using the present PS/QDs films with those of PbS/CdS QDs is difficult, because of core shell structure in the later. In such situation the charge carrier transfer is mediated by energy states introduced by broad energy band of $\mathrm{CdS}$ shell. It was reported that depending on the thickness, the presence of CdS core strongly influences the processes of charge carriers transfer between $\mathrm{PbS}$ and its surrounding, as well as it strongly affects the trap states on the surface of PbS NCs. ${ }^{[1,44-45]}$

Table 1 and Figure 2 show that the increase of $k_{h}$ value with the size of the QDs is almost linear while the dependence of $\mathrm{k}_{\mathrm{e}}$ strongly deviates from linearity. The change in the 
size of QDs from 2.9 to $4.5 \mathrm{~nm}$ lead to an increase of $\mathrm{k}_{\mathrm{e}}$ from $0.1 \times 10^{10} \mathrm{~s}^{-1}$ to $2.17 \times 10^{10} \mathrm{~s}^{-1}$, which remains the same for QDs with diameter of $5.3 \mathrm{~nm}$. This behavior points out the occurrence of a different change in the PS/QDs systems that significantly influences one kind of recombining charge carriers. Figure 1f shows that the change of the QDs diameter results in an appearance of a new deactivation channel for electrons. The CB energy level in QDs becomes lower than that of PS (Scheme 1b). For QDs of $4.5 \mathrm{~nm}$ and $5.3 \mathrm{~nm}$, the transition between $\mathrm{CBs}$ of PS and of PbS become possible speeding up the process of electron transfer. Surprisingly, the results show that $k_{\mathrm{e}}>k_{\mathrm{h}}$ even for the systems where the electron transfer to $\mathrm{CB}$ of QDs is not possible (size: 2.9 and $3.2 \mathrm{~nm}$ ), and in which the driving force for the hole transfer should be smaller than that for electron. This result indicates that both electron and hole transfers might be mediated by the interface trap states. A possible explanation is that the density of electron trap states in PS/QDs interface is much larger than that of hole trap states, hence the transition of electrons to QDs is faster than the process for holes. If the density of surface transition channels is lower for holes, these charge carriers might be accumulated at the interface, and slowly transferred to the VB of QDs. Strong effect of trap-states on the dynamics of charge carriers transfer in the systems containing semiconductor materials were previously reported. ${ }^{[43,46-47]}$

As we have already discussed, the $k_{\mathrm{e}}$ and $k_{\mathrm{h}}$ components reflect two processes: charge carriers diffusion within the PS domain, and transfer at the interface of both materials. Figure $2 \mathrm{~b}$ shows that electron transfer rate constant is the same for the systems which contain QDs of 4.5 and $5.3 \mathrm{~nm}$. As the distance between the nanostructures in these two systems is similar ( $25 \mathrm{~nm}$ ), the contribution of electron diffusion ( $\mathrm{k}_{\text {ediff }}$ ) to the processes of electron deactivation $\left(\mathrm{k}_{\mathrm{e}}\right)$ are the same in both samples. However, for the systems containing QDs of different sizes the velocity of electron transfer at the interfaces should be different, due to different number and reduced energies of the electronic states in the nanostructures. This is in agreement with 
the holes behavior in all PS/QD systems and with the electron behavior in the PS containing QDs of smaller sizes. The increasing difference between the energies of CBs in PS and QDs should speed up the process of electron transfer at the interface, due to faster depopulation of the initially populated trap states in $\mathrm{PbS}$ QDs. As a result, we should see larger value of $\mathrm{k}_{\mathrm{e}}$ for the sample containing QDs of $5.3 \mathrm{~nm}$ as compared to the system with QDs of $4.5 \mathrm{~nm}$. Similar values of $\mathrm{k}_{\mathrm{e}}$ in both systems suggest that the electron transfer $\left(\mathrm{k}_{\mathrm{et}}\right)$ time is (i) the same in both cases, or (ii) it is different but shorter than the temporal resolution of the experiment ( $70 \mathrm{fs})$. The first possibility is in contradiction with the observed correlation between $\mathrm{k}_{\mathrm{h}}$ for all PS/QDs systems and $k_{\mathrm{e}}$ for smaller QDs as well as with our previous results ${ }^{[16]}$ Therefore, the second option has to be taken into account. From the experimental point of view, similar values of $k_{e}$ in both systems mean that the times of electron transfer $\left(\tau_{\mathrm{et}}\right)$ at the interface are different for both samples, but they are below the temporal resolution of the setup. In this

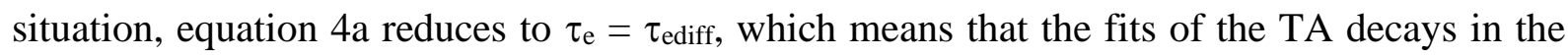
systems containing QDs of 4.5 and $5.3 \mathrm{~nm}$ will give the same values of $k_{\mathrm{e}}=1 / \tau_{\text {ediff }}=$ $2.17 \times 10^{10} \mathrm{~s}^{-1}$.

This information in combination with the knowledge of the electron and hole mobilities in PS and PS/QDs allow us to estimate the values of charge carrier's diffusion time constants in the PS films that contain QDs of different diameters (Table 2). Recent reports indicate balanced electron $\left(12.5 \mathrm{~cm}^{2} \mathrm{~V}^{-1} \mathrm{~s}^{-1}\right)$ and hole mobilities $\left(7.5 \mathrm{~cm}^{2} \mathrm{~V}^{-1} \mathrm{~s}^{-1}\right)$ in $\mathrm{MAPbI}_{3}$ thin polycrystalline films, in agreement with theoretical predictions, giving similar effective masses of those charges $\left(\mathrm{m}_{\mathrm{e}}=0.23 \mathrm{~m}_{0}\right.$ and $\left.\mathrm{m}_{\mathrm{h}}=0.29 \mathrm{~m}_{0}\right){ }^{[48-50]}$ The THz measurements of charge carrier mobilities $(\mu)$ in $\mathrm{MAPbI}_{3}$ have given the values $\mu_{\mathrm{e}}=2 \mu_{\mathrm{h}}{ }^{[50-51]}$ If the mobility of charge carriers within PS is not affected by the presence of QDs, the diffusion rate constant of the electrons ( $\mathrm{k}_{\text {ediff }}$ ) would be two times larger than that of the holes ( $\mathrm{k}_{\text {hdiff }}$ ). In fact, the comparison of THz signals in neat PS and PS/QDs indicates similar initial mobilities $\left(20 \mathrm{~cm}^{2}\right.$ 
$\mathrm{V}^{-1} \mathrm{~s}^{-1}$ ), and thus the diffusion in all samples depends mainly on the PS nature (Figure 3).As the distances between the QDs in the nanocomposites are similar $(21-25 \mathrm{~nm})$, the electrons and holes diffusion constants of all samples should be the same independently on the size of QDs. Hence, the value of $k_{\text {ediff }}\left(2.17 \times 10^{10} \mathrm{~s}^{-1}\right)$ in PS domain containing QDs of 4.5 and $5.3 \mathrm{~nm}$ is similar to those of all PS/QDs systems. Because $\mu_{\mathrm{e}}=2 \mu_{\mathrm{h}}$, the value of $\mathrm{k}_{\text {hdiff }}\left(1.09 \times 10^{10} \mathrm{~s}^{-1}\right)$ will be two times smaller than kediff.

Next, the above extracted diffusion rate constants of electrons and holes allow to calculate the values of transition rate constants of charge carriers $\left(k_{\text {et }}\right.$ and $\left.k_{h t}\right)$ in all PS/QDs systems (Table 2). The resulted values show that the transition of holes is much slower than that of electrons. Moreover, the total transfer rate constants of holes ( $\mathrm{k}_{\mathrm{ht}}$ ) are dominated by component ascribed to transition to the $\mathrm{CB}$ of $\mathrm{PbS}$ QDs. Using $k_{\text {ediff }}$ and $k_{\text {hdiff }}$ (Table 2) along with the total mobility $\left(\mu=\mu_{\mathrm{e}}+\mu_{\mathrm{h}}\right)$, the total diffusion length of charge carriers can be also calculated using the equation: ${ }^{[23]}$

$$
L=\sqrt{\frac{\mu k_{b} T}{e\left(k_{\text {ediff }}+k_{\text {hdiff }}\right)}},
$$

Where $k_{\mathrm{b}}$ is the Boltzmann constant, $T$ is the temperature of the sample and e relates to the elemental charge. The calculated $\mathrm{L}$ is equal to $39 \mathrm{~nm}$ (Table 2) and it is approximately two times longer than the mean distance between QDs of different size embedded in PS (21 - 25 $\mathrm{nm})$. The difference is because the calculated value is related to the diffusion length of both electrons and holes.

\subsection{Pump fluence dependence of charge carriers dynamics}

Important information of charge carrier generation, cooling as well as recombination/transfer processes, can be extracted from exploring charge carrier dynamics upon increasing the pump fluence. Firstly, we discuss the results of the dependence of photoinduced TA signals on the fluence of the absorbed photons ranging from $8.2 \times 10^{12}$ to 
$1.23 \times 10^{15} \mathrm{ph} / \mathrm{cm}^{2}$ for neat PS and PbS NCs of $3.2 \mathrm{~nm}$ embedded in PS film at 2 ps pump-probe delay (Figure 4a,b). Clearly, the increase in the pump fluence results in a broadening of PB band with a maximum intensity at $755 \mathrm{~nm}$, and the appearance of a new sub-band at 730/740 $\mathrm{nm}$ for both samples. Moreover, in the case of PS/QDs, the new band (at $740 \mathrm{~nm}$ ) shows a stronger TA signal than the one in neat film of PS $(755 \mathrm{~nm})$. Recent TA experiments on PS films indicated a broadening of the bleach band and its small shift at early times upon increasing the fluence of the pump due to a band filling. ${ }^{[36,52-53]}$ However, the appearance of an additional band was not observed, and thus one can distrust the observed behavior. Previous experiments on perovskite films were carried out at different lower fluences (1-100

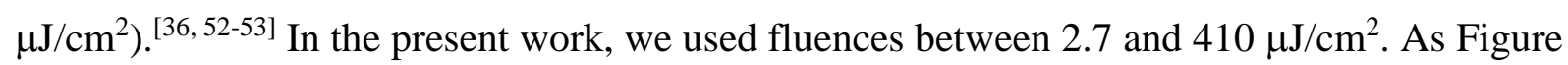
4a shows, the bleach band at $760 \mathrm{~nm}$ for PS film in the range of used fluences in previous reports is characterized by one band. The change is only a spectral broadening of the bleach band and a small shift of its minimum which agrees with state filling model. New bands in PS appear upon pumping with fluences significantly higher than $100 \mu \mathrm{J} / \mathrm{cm}^{2}$. Under these experimental conditions, periodic features appear in the TA spectra of both PS and PS/QDs (Figure 5a). One possible reason of such behavior is an interference of a probe light in thin PS layer. However, we checked that the change of the relative angle between probe beam and surface of the samples does not affect the observed features, and thus the modulation by the interference has to be excluded. Additionally, the TA spectra of PS and PS/QDs recorded at the lowest fluence do not show any periodic modulations (Figure S4). The comparison of the TA spectra of PS and PS/QDs films at higher energies clearly shows the existence of additional bands which disappear at approximately 20 ps pump-probe delay time (Figure S5). Furthermore, the periodic structure is absent in the TA spectra at longer pump-probe delays, excluding interferences in thin layer as the origin of the periodicities: in a such case, we expect the modulation of a weak positive signal visible at shorter wavelengths for pump-probe 
delays longer than 20 ps. The presence of new sub-bands reflects the appearance of new electronic transitions in the studied systems, and it is consistent with the results reported for neat PS upon excitation with high fluences. ${ }^{[24]}$ The absorption of a high density of photons leads to the saturation of the excited energy states, and to bleaching of the interband optical transition. The excited charge carriers become a source of new local electric field which, in turn, tends to shift the optical transitions and changes their oscillator strengths. ${ }^{[22,54]}$ Additionally, high initial charge carriers concentration may result in the generation of new transitions at energies spectrally shifted in respect to the low-excitation transitions. ${ }^{[55]}$ Moreover, the increase in the density of the initially photoexcited charge carriers results in more efficient formation of excitons.

Recently, efficient formation of electron-hole couples bound by Coulomb forces was reported for $\mathrm{FAPbI}_{3}{ }^{\left[{ }^{[24]}\right.}$ Opposite to free charge carriers, the ultrafast decay of the excitons population was observed ( 5 ps). ${ }^{[24,56-57]}$ Figures S6 show TA decays in neat PS and PS/QDs systems at representative observation wavelengths within the bleaching band. The new subbands decay within the first 20 ps (Figure S6). Unfortunately, due to the complex nature of transition induced by the high density of photons in the pump beam, we could not apply our model to fit this decay. However, the observed temporal characteristic of the TA decay is similar to that reported for $\mathrm{FAPbI}_{3}$, and thus it is more probably attributed to exciton population. ${ }^{[24]}$ Interestingly, upon excitation at higher pump fluences, the bleaching bands of both samples at $1 \mathrm{~ns}$ pump-probe delay time shows that the band with the maximum intensity at 730/740 nm still exists (Figure S7e,f). Not all populations observed at shorter wavelengths decay within 20 ps. As the density of the excited charge carriers in PS is much lower due to the processes of recombination, diffusion and additionally transition to QDs (in the case of PS/QDs), the observed feature cannot be related to the interactions between high densities of electrons and holes. This suggests that at a high fluence of the absorbed photons may result in 
the formation and population of excitonic traps within PS and in the PS/QDs interface. The presence of the additional signal might be related to the degradation of the sample due to a high density of the absorbed photons. In order to exclude this possibility, the sample was moved during the measurement. Moreover, the experiments were repeated several times giving similar results.

To check the formation efficiency of new transitions, we fitted the bleaching bands of neat PS and PS/QDs films by Lorentz functions (Figures S7 and S8). Homogeneous spectral broadening in PS, due to strong phonon coupling of charge carriers arising from their polaronic properties was reported. ${ }^{[58-59]}$ We have two observations. Firstly, the results indicate that the maximum positions of photogenerated sub-bands in both samples do not depend on the fluence of the absorbed photons. Secondly, the comparison of the integral intensities of the two sub-bands with intensity maxima at $755 \mathrm{~nm}$ and 730/740nm (neat PS and PS/QDs, respectively) at 2 ps pump-probe delay time indicates different dependence of peak intensities on the pump fluences (Figures S7 and S8).

For the few first fluences, only the band at $755 \mathrm{~nm}$ in PS is present, while the $730 \mathrm{~nm}$ one appears at a fluence of $4.1 \times 10^{14} \mathrm{ph} / \mathrm{cm}^{2}$, and it does not change its relative intensity. For PS/QDs film, the $740 \mathrm{~nm}$ band is absent only at the lowest pump fluence and its relative intensity rises with increasing fluence of the absorbed photons. Moreover, the intensity of the signal at $740 \mathrm{~nm}$ is much higher than that at $755 \mathrm{~nm}$. The $730 \mathrm{~nm}$ sub-band in neat PS is still present at $1 \mathrm{~ns}$ pump-probe delay time upon excitation at the highest energies, but its intensity is weak (Figures 5c, S7 and S8). On the other hand, in PS/QDs film the integral intensities of the signals at 740 and at $755 \mathrm{~nm}$ at $1 \mathrm{~ns}$ have comparable values (Figure S8). For the origin of PB upon excitation at high fluences, we assign the signal at $730 / 740 \mathrm{~nm}$ to excitons, and points out that these populations decay within the first 20 ps which is apparently incompatible with the presence of these feature at $1 \mathrm{~ns}$ pump-probe delay time. As we mentioned above, 
increasing the density of photoexcited charge carriers leads to higher concentration of excitons, and thus the probability of excitonic trap population increases.[24,28,60] Trapped excitons can survive for relatively long time, contributing to the TA signal. Previous reports on $\mathrm{MAPbI}_{3}$ indicated the presence of both bulk and surface excitonic traps and the occurrence of excitons trapping within picoseconds. ${ }^{[61-62]}$ The QDs embedded in PS matrix might result in an increase of excitonic traps density formed in the PS/QDs interface. ${ }^{[16]}$ As a result, the probability of rapid trapping of initially photoexcited excitons rises, and thus the presence and the high intensity of the signal at $740 \mathrm{~nm}$ in PS/QDs.

Application of photovoltaic materials in commercial devices such as LEDs requires low non-radiative recombination and efficient charge carriers transport. Consequently, to fulfill these requirements the processes of electron and holes trapping must be reduced. In this report, we show a clear PbS QDs size effect on the ultrafast dynamics of electrons and holes in PS/QDs materials applicable to NIR-LEDs. Our results show ultrafast and efficient charge carriers transfer from perovskite matrix to $\mathrm{PbS}$, which speeds up with the size of QDs. The acceleration of such processes decreases the contribution of undesirable charge carriers trapping and non-radiative recombination within PS. Owing to this fact, our result shows a way to increase the efficiency of perovskite/PbS QDs films-based LEDs.

\section{Conclusions}

For the first time, we demonstrate a strong correlation between deactivation processes of photoexcited charge carriers in PS and the size of PbS QDs embedded in its matrix. The increase of the QDs size results in an acceleration of electron and hole transition from PS to the QDs. Our kinetic model allows to extract the total transfer rate constants of electrons $\left(\mathrm{k}_{\mathrm{e}}\right)$ and holes $\left(\mathrm{k}_{\mathrm{h}}\right)$ in the studied systems. The values of $\mathrm{k}_{\mathrm{e}}$ and $\mathrm{k}_{\mathrm{h}}$ increase from $0.1 \mathrm{~s}^{-1}$ and $1 \times 10^{9}$ $\mathrm{s}^{-1}$ to $4.5 \mathrm{~s}^{-1}$ and $22 \times 10^{9} \mathrm{~s}^{-1}$ with the size of QDs increasing from $2.9 \mathrm{~nm}$ to $5.3 \mathrm{~nm}$, respectively. This is due to the increasing concentration and changes in the energies of the 
QDs electronic states. Moreover, taking into account that the charge carrier diffusion in the PS matrix does not depend on the presence of QDs, we show that the processes of electron and hole transfer to QDs are mediated by PS/QDs interfacial trap states. The analysis of the correlation between the size of QDs and the resulted electron and hole recombination rate constants along with the value of charge carrier's mobility in PS and PS/QDs systems allow to estimate the electron and hole diffusion rate constants $\left(k_{\text {ediff }}=2.2 \times 10^{10} \mathrm{~s}^{-1}\right.$ and $k_{\text {hdiff }}=1.1 \times$ $10^{10} \mathrm{~s}^{-1}$, respectively). Due to similar distance between QDs within PS matrix regardless of the different QD sizes analyzed, these values are similar for all PS/QDs nanocomposites. The transfer rate constants of electrons ( $\left.\mathrm{k}_{\mathrm{et}}\right)$ and holes $\left(\mathrm{k}_{\mathrm{ht}}\right)$ increase with the size of QDs $(0.1$ to 1.6 $\left.\times\left(10^{10} \mathrm{~s}^{-1}\right)\right)$ and $\left(0.1\right.$ to $\left.0.8 \times\left(10^{10} \mathrm{~s}^{-1}\right)\right)$, respectively. In the case of 4.5 and $5.3 \mathrm{~nm}$ QDs, the time of the electron transfer at the interface is shorter than $70 \mathrm{fs}$, the temporal resolution of the setup. The power-dependents spectral behavior of PS and PS/QDs systems suggests the increasing contribution of excitons in the photoinduced dynamics upon pumping with higher pump fluences. We also show that the survival probability of the excitonic state is higher in the case of PS/QDs systems than in neat PS which proves a higher concentration of excitonic states in the PS/QDs interface than in PS matrix. This fact could have important implications in the performance of optoelectronic devices based on this kind of hybrid material. The results shown here provide new insights into the ultrafast processes occurring in $\mathrm{PS} / \mathrm{PbS}$ QDs systems and the relevance of the QDs size in the electrons, holes and excitons dynamics. These results could have important implications to increase the efficiency of nanocompositebased LED.

\section{Materials and methods}

The $\mathrm{PbS}$ QDs of different sizes were synthesized and dispersed in an octane solution following the method described in detail in the Supporting Information. ${ }^{[3-4,25]}$ The QDs properties were characterized by Transmission Electron Microscopy (TEM) and 
photoluminescence (PL) spectroscopy was carried out before and after the ligand exchange with the halide perovskite (PS) precursors, MAI and $\mathrm{PbI}_{2}$, see Figure $\mathrm{S} 9$ and $\mathrm{S} 10 .{ }^{[26]}$ In order to produce layers with embedded $\mathrm{PbS}$ QDs, ligand exchange is necessary to make $\mathrm{PbS}$ QDs soluble into DMF solution with the PS precursors used for the preparation of thin films. Figure $\mathrm{S} 11$ shows that ligand exchange, where oleic acid capping is substituted by a $\mathrm{MAPbI}_{3}$ shell, is successfully carried out as the signature of oleic acid disappears from the FTIR spectra. ${ }^{[63]}$ The thin films with embedded $\mathrm{PbS}$ QDs present $\mathrm{MAPbI}_{3}$ tetragonal structure, see Figure S12, ${ }^{[64]}$ thus indicating that PS is formed independently of the presence of PbS QDs that in fact have a seeding effect as previously reported. ${ }^{[3,7,18]}$ See Experimental Section at Supporting Information for more details.

Thin films of $\mathrm{MAPbI}_{3}$ with embedded $\mathrm{PbS}$ QDs have been analyzed with timeintegrated absorption, fs-time-resolved Vis-NIR transient absorption, terahertz and nanosecond flash photolysis techniques (described in detail in the Experimental Section at the Supporting Information).In order to evaluate the effect of the QD size, four different sizes 2.9, 3.2, 4.5 and $5.3 \mathrm{~nm}$ were used at similar concentrations of nanoparticles per cubic centimeter of PS host matrix.

\section{Acknowledgements:}

This work was support by the MINECO (Spain) through project MAT-2017-8653-R, Generalitat Valenciana via Prometeo Grant Q-Devices (Prometeo/2018/098), and the European Research Council (ERC) via Consolidator Grant (724424-No-LIMIT).PP acknowledges the support from National Science Centre (Poland) through project No. 2017/26/D/ST3/00910. 


\section{Appendix A. Supplementary material}

Supplementary data associated with this article can be found in the online version at https://www.journals.elsevier.com/nano-energy 


\section{References}

[1] Z. Huang, Z. Xu, M. Mahboub, X. Li, J. W. Taylor, W. H. Harman, T. Lian, M. L. Tang, Angew. Chem. Int. Ed. 2017, 56, 16583.

[2] T. T. Ngo, I. Mora-Sero, J. Phys. Chem. Lett. 2019, 10, 1099.

[3] T. T. Ngo, I. Suarez, R. S. Sanchez, J. P. Martinez-Pastor, I. Mora-Sero, Nanoscale 2016, 8, 14379

[4] R. S. Sanchez, M. S. de la Fuente, I. Suarez, G. Munoz-Matutano, J. P. MartinezPastor, I. Mora-Sero, Sci. Adv. 2016, 2, 1501104.

[5] X. Gong, Z. Yang, G. Walters, R. Comin, Z. Ning, E. Beauregard, V. Adinolfi, O. Voznyy, E. H. Sargent, Nat. Photon. 2016, 10, 253;

[6] Z. Yang, O. Voznyy, G. Walters, J. Z. Fan, M. Liu, S. Kinge, S. Hoogland, E. H. Sargent, ACS Photonics 2017, 4, 830.

[7] S. S. Li, C. H. Chang, Y. C. Wang, C. W. Lin, D. Y. Wang, J. C. Lin, C. C. Chen, H. S. Sheu, H. C. Chia, W. R. Wu, U. S. Jeng, C. T. Liang, R. Sankar, F. C. Chou, C. W. Chen, Energy Environ. Sci. 2016, 9, 1282.

[8] J. Han, S. Luo, X. Yin, Y. Zhou, H. Nan, J. Li, X. Li, D. Oron, H. Shen, H. Lin, Small 2018, 14, 1801016;

[9] T. T. Ngo, S. Masi, P. F. Mendez, M. Kazes, D. Oron, I. M. Sero, Nanoscale Adv. 2019, $1,4109$.

[10] X. P. Zheng, J. Troughton, N. Gasparini, Y. B. Lin, M. Y. Wei, Y. Hou, J. K. Liu, K. P. Song, Z. L. Chen, C. Yang, B. Turedi, A. Y. Alsalloum, J. Pan, J. Chen, A. A. Zhumekenov, T. D. Anthopoulos, Y. Han, D. Baran, O. F. Mohammed, E. H. Sargent, O. M. Bakr, Joule 2019, 3, 1963; 
[11] J. Z. Fan, N. T. Andersen, M. Biondi, P. TodoroviC, B. Sun, O. Ouellette, J. Abed, L. K. Sagar, M. J. Choi, S. Hoogland, F. P. G. de Arguer, E. H. Sargent, Adv. Mater. 2019, 904304

[12] Z. Moradi, H. Fallah, M. Hajimahmoodzadeh, Sens. Actuator A-Phys. 2018, 280, 47;

[13] C. Liu, H. Peng, K. Wang, C. Wei, Z. Wang, X. Gong, Nano Energy 2016, 30, 27.

[14] Z. Y. Yang, A. Janmohamed, X. Z. Lan, F. P. G. de Arquer, O. Voznyy, E. Yassitepe, G. H. Kim, Z. J. Ning, X. W. Gong, R. Comin, E. H. Sargent, Nano Lett. 2015, 15, 7539;

[15] M. X. Liu, O. Voznyy, R. Sabatini, F. P. G. de Arquer, R. Munir, A. H. Balawi, X. Z. Lan, F. J. Fan, G. Walters, A. R. Kirmani, S. Hoogland, F. Laquai, A. Amassian, E. H. Sargent, Nat. Mater. 2017, 16, 258.

[16] P. Galar, P. Piatkowski, T. T. Ngo, M. Gutierrez, I. Mora-Sero, A. Douhal, Nano Energy 2018, 49, 471.

[17] R. S. Sanchez, E. Binetti, J. A. Torre, G. Garcia-Belmonte, M. Striccoli, I. Mora-Sero, Nanoscale 2014, 6, 8551;

[18] C.-Y. Lin, S.-S. Li, J.-W. Chang, H.-C. Chia, Y.-Y. Hsiao, C.-J. Su, B.-J. Lian, C.-Y. Wen, S.-K. Huang, W.-R. Wu, D.-Y. Wang, A.-C. Su, C.-W. Chen, U. S. Jeng, Adv. Funct. Mater. 2019, 29, 1902582.

[19] S. Masi, C. Echeverria-Arrondo, S. K. P. Muhammed, T. T. Ngo, P. F. Mendez, E. López-Fraguas, D. F. Macias-Pinilla, J. Planelles, J. I. Climente, I. Mora-Seró, ACS Energy Lett. 2020, 5, 418.

[20] A. P. Alivisatos, Science 1996, 271, 933.

[21] M. J. Choi, Y. J. Kim, H. Lim, E. Alarousu, A. Adhikari, B. S. Shaheen, Y. H. Kim, O. F. Mohammed, E.. H. Sargent, J. Y. Kim, Y. S. Jung, s. Adv. Mater. 2019, 1805886

[22] P. Piatkowski, B. Cohen, F. J. Ramos, M. Di Nunzio, M. K. Nazeeruddin, M. Graetzel, S. Ahmad, A. Douhal, Phys. Chem. Chem. Phys. 2015, 17, 14674. 
[23] P. Piatkowski, B. Cohen, C. S. Ponseca, Jr., M. Salado, S. Kazim, S. Ahmad, V. Sundstrom, A. Douhal, J. Phys. Chem. Lett. 2016, 7, 204.

[24] P. Piatkowski, B. Cohen, S. Kazim, S. Ahmad, A. Douhal, Phys. Chem. Chem. Phys. 2016, 18, 27090.

[25] Z. J. Ning, X. W. Gong, R. Comin, G. Walters, F. J. Fan, O. Voznyy, E. Yassitepe, A. Buin, S. Hoogland, E. H. Sargent, Nature 2015, 523, 324.

[26] I. Moreels, K. Lambert, D. Smeets, D. De Muynck, T. Nollet, J. C. Martins, F. Vanhaecke, A. Vantomme, C. Delerue, G. Allan, Z. Hens, ACS Nano 2009, 3, 3023.

[27] H. Beygi, S. A. Sajjadi, A. Babakhani, J. F. Young, F. van Veggel, Appl. Surf. Sci. $\mathbf{2 0 1 8}, 457,1$

[28] H.-S. Kim, C.-R. Lee, J.-H. Im, K.-B. Lee, T. Moehl, A. Marchioro, S.-J. Moon, R. Humphry-Baker, J.-H. Yum, J. E. Moser, M. Graetzel, N.-G. Park, Sci. Rep. 2012, 2.

[29] J. Jasieniak, M. Califano, S. E. Watkins, ACS Nano 2011, 5, 5888.

[30] B. R. Hyun, Y. W. Zhong, A. C. Bartnik, L. F. Sun, H. D. Abruna, F. W. Wise, J. D. Goodreau, J. R. Matthews, T. M. Leslie, N. F. Borrelli, ACS Nano 2008, 2, 2206.

[31] G. Xing, N. Mathews, S. Sun, S. S. Lim, Y. M. Lam, M. Graetzel, S. Mhaisalkar, T. C. Sum, Science 2013, 342, 344.

[32] H.-Y. Hsu, C.-Y. Wang, A. Fathi, J.-W. Shiu, C.-C. Chung, P.-S. Shen, T.-F. Guo, P. Chen, Y.-P. Lee, E. W.-G. Diau, Angew. Chem. -Int. Ed. 2014, 53, 9339.

[33] C. Wehrenfennig, G. E. Eperon, M. B. Johnston, H. J. Snaith, L. M. Herz, Adv. Mater. 2014, 26, 1584;

[34] L. M. Herz, in Annu. Rev. Phys.Chem., (Eds: M. A. Johnson, T. J. Martinez), 2016, 67,65 .

[35] Z. Guo, J. S. Manser, Y. Wan, P. V. Kamat, L. Huang, Nat. Commun. 2015, 6, 7471.

[36] J. S. Manser, P. V. Kamat, Nat. Photonics 2014, 8, 737. 
[37] M. B. Johnston, L. M. Herz, Acc. .Chem. Res. 2016, 49, 146.

[38] J. Huang, Y. Yuan, Y. Shao, Y. Yan, Nat. Rev. Mater. 2017, 2, 17042.

[39] E. M. Hutter, J. J. Hofman, M. L. Petrus, M. Moes, R. D. Abellon, P. Docampo, T. J. Savenije, Adv. Energy Mater. 2017, 1602349

[40] G. H. Carey, A. L. Abdelhady, Z. J. Ning, S. M. Thon, O. M. Bakr, E. H. Sargent, Chem. Rev. 2015, 115, 12732.

[41] K. Tvrdy, P. A. Frantsuzov, P. V. Kamat, Proc. Natl. Acad. Sci.U.S.A. 2011, 108, 29.

[42] A. J. Ward, A. Ruseckas, M. M. Kareem, B. Ebenhoch, L. A. Serrano, M. Al-Eid, B. Fitzpatrick, V. M. Rotello, G. Cooke, I. D. W. Samuel, Adv. Mater. 2015, 27, 2496.

[43] J. H. Olshansky, A. D. Balan, T. X. Ding, X. Fu, Y. V. Lee, A. P. Alivisatos, ACS Nano 2017, 11, 8346.

[44] H. Zhao, Z. Fan, H. Liang, G. S. Selopal, B. A. Gonfa, L. Jin, A. Soudi, D. Cui, F. Enrichi, M. M. Natile, I. Concina, D. Ma, A. O. Govorov, F. Rosei, A. Vomiero, Nanoscale 2014, 6, 7004;

[45] H. D. Zang, P. K. Routh, Q. P. Meng, M. Cotlet, Nanoscale 2017, 9, 14664.

[46] A. J. Harvie, C. T. Smith, R. Ahumada-Lazo, L. J. C. Jeuken, M. Califano, R. S. Bon, S. J. O. Hardman, D. J. Binks, K. Critchley, J. Phys. Chem. C 2018, 122, 10173.

[47] A. Pal, S. Srivastava, R. Gupta, S. Sapra, Phys. Chem. Chem. Phys. 2013, 15, 15888.

[48] Y. H. Chang, C. H. Park, K. Matsuishi, J. Korean Phys. Soc. 2004, 44, 889;

[49] G. Giorgi, J. I. Fujisawa, H. Segawa, K. Yamashita, J. Phys. Chem. Lett. 2013, 4, 4213.

[50] C. S. Ponseca, Jr., T. J. Savenije, M. Abdellah, K. Zheng, A. Yartsev, T. Pascher, T. Harlang, P. Chabera, T. Pullerits, A. Stepanov, J.-P. Wolf, V. Sundstrom, J. Am. Chem. Soc. 2014, 136, 5189. 
[51] C. S. Ponseca, Jr., E. M. Hutter, P. Piatkowski, B. Cohen, T. Pascher, A. Douhal, A. Yartsev, V. Sundstrom, T. J. Savenije, J. Am. Chem. Soc. 2015, 137, 16043.

[52] K. Pydzinska-Bialek, J. Szeremeta, K. Wojciechowski, M. Ziolek, J. Phys. Chem. C 2019, 123, 110.

[53] T. C. Sum, N. Mathews, G. C. Xing, S. S. Lim, W. K. Chong, D. Giovanni, H. A. Dewi, Acc. Chem. Res. 2016, 49, 294.

[54] A. A. Mikhailovsky, A. V. Malko, J. A. Hollingsworth, M. G. Bawendi, V. I. Klimov, Appl. Phys.Lett. 2002, 80, 2380.

[55] Ed. V. I. Klimov, Taylor \& Francis, New York, ch. 5 2005, 200-208.

[56] H. Wang, L. Whittaker-Brooks, G. R. Fleming, J. Phys. Chem. C 2015, 119, 19590;

[57] C. Sheng, C. Zhang, Y. Zhai, K. Mielczarek, W. Wang, W. Ma, A. Zakhidov, Z. V. Vardeny, Phys. Rev. Lett. 2015, 114, 116601.

[58] C. Wehrenfennig, M. Z. Liu, H. J. Snaith, M. B. Johnston, L. M. Herz, J. Phys. Chem. Lett. 2014, 5, 1300;

[59] D. B. Straus, S. H. Parra, N. Iotov, J. Gebhardt, A. M. Rappe, J. E. Subotnik, J. M. Kikkawa, C. R. Kagan, J. Am. Chem. Soc. 2016, 138, 13798.

[460] M. Saba, M. Cadelano, D. Marongiu, F. Chen, V. Sarritzu, N. Sestu, C. Figus, M. Aresti, R. Piras, A. G. Lehmann, C. Cannas, A. Musinu, F. Quochi, A. Mura, G. Bongiovanni, Nat. Commun. 2014, 5, 5049 .

[61] J. J. Peng, Y. N. Chen, K. B. Zheng, T. Pullerits, Z. Q. Liang, Chem. Soc. Rev. 2017, 46, 5714;

[62] X. Wu, M. T. Trinh, D. Niesner, H. Zhu, Z. Norman, J. S. Owen, O. Yaffe, B. J. Kudisch, X. Y. Zhu, J. Am. Chem. Soc. 2015, 137, 2089.

[63] D. Zherebetskyy, M. Scheele, Y. J. Zhang, N. Bronstein, C. Thompson, D. Britt, M. Salmeron, P. Alivisatos, L. W. Wang, Science 2014, 344, 1380. 
[64] N. J. Jeon, J. H. Noh, Y. C. Kim, W. S. Yang, S. Ryu, S. I. Seok, Nat. Mater. 2014, $13,897$.

\begin{tabular}{ccccccc}
\hline $\begin{array}{c}\text { QD size } \\
(\mathbf{n m})\end{array}$ & $\begin{array}{c}\boldsymbol{k}_{\mathbf{h}} \\
\left(\mathbf{1 0}^{\mathbf{9}} \mathbf{s}^{\mathbf{- 1}}\right)\end{array}$ & $\begin{array}{c}\boldsymbol{k}_{\mathbf{e}} \\
\left(\mathbf{1 0}^{\mathbf{1 0}} \mathbf{s}^{\mathbf{- 1}}\right)\end{array}$ & $\begin{array}{c}\boldsymbol{k}_{\mathbf{e x}} \\
\left(\mathbf{1 0}^{\mathbf{1 3}} \mathbf{s}^{-\mathbf{1}}\right)\end{array}$ & $\boldsymbol{P}_{\mathbf{1}}(\boldsymbol{\%})$ & $\boldsymbol{P}_{\mathbf{2}}(\boldsymbol{\%})$ & $\boldsymbol{n}_{\mathbf{e x}}(\boldsymbol{\%})$ \\
\hline $\mathbf{2 . 9}$ & 0.95 & 0.10 & 2.48 & 77 & 23 & - \\
$\mathbf{3 . 2}$ & 1.59 & 0.89 & 1.94 & 91 & 9 & - \\
$\mathbf{4 . 5}$ & 3.52 & 2.17 & 0.85 & 92 & 8 & 1 \\
$\mathbf{5 . 3}$ & 4.48 & 2.18 & 1.77 & 97 & 2 & 1 \\
\hline
\end{tabular}

Table 1. Values of rate constants for holes, electrons, and excitons, and population fractions within areas affected $\left(\mathrm{P}_{1}\right)$ and not affected $\left(\mathrm{P}_{2}\right)$ by the presence of QDs. The data are obtained using thekinetic model described in the text to fit the transient absorption decays of $\mathrm{MAPbI}_{3} / \mathrm{QDs}$ film, containing PbS crystals of different sizes.

\begin{tabular}{cccc}
\hline $\begin{array}{c}\text { QD size } \\
(\mathbf{n m})\end{array}$ & $\begin{array}{c}\boldsymbol{k}_{\mathbf{h t}} \\
\left(\mathbf{1 0}^{\mathbf{9}} \mathbf{s}^{-}\right. \\
\mathbf{1})\end{array}$ & $\begin{array}{c}\boldsymbol{k}_{\text {et }} \\
\left(\mathbf{1 0}^{\mathbf{1 0}} \mathbf{s}^{-\mathbf{1}}\right)\end{array}$ & $\begin{array}{c}\boldsymbol{L} \\
(\mathbf{n m})\end{array}$ \\
\hline $\mathbf{2 . 9}$ & 1.04 & 0.11 & 39 \\
$\mathbf{3 . 2}$ & 1.86 & 1.56 & 39 \\
$\mathbf{4 . 5}$ & 5.32 & $>1430^{(\mathrm{a})}$ & 39 \\
$\mathbf{5 . 3}$ & 7.81 & $>1430^{(\mathrm{a})}$ & 39 \\
\hline
\end{tabular}

Table 2. Values of rate constants of electrons and holes diffusion and transfer in PS/QDs samples having $\mathrm{PbS}$ of the indicated size. (a) estimated based on our time resolution $70 \mathrm{fs}$ $\left(\mathrm{k}_{\mathrm{et}}>\left(70 \times 10^{-15} \mathrm{~s}\right)^{-1}\right)$. 


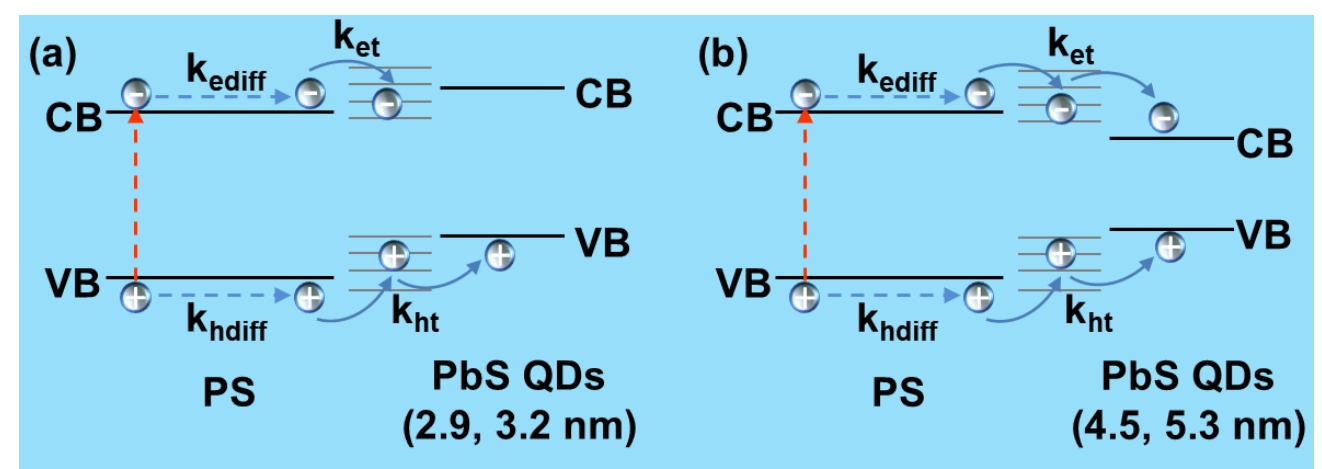

Scheme 1. Scenarios of photoinduced hole and electron diffusion and transfer to QDs of different sizes. (a) The electron transfer to the CB in QDs of 2.9 and $3.2 \mathrm{~nm}$ is precluded. (b) The electron transfer to the CB of QDs of 4.5 and $5.3 \mathrm{~nm}$ is allowed. In both scenarios the hole can be transferred from the PS VB to the QD one. 

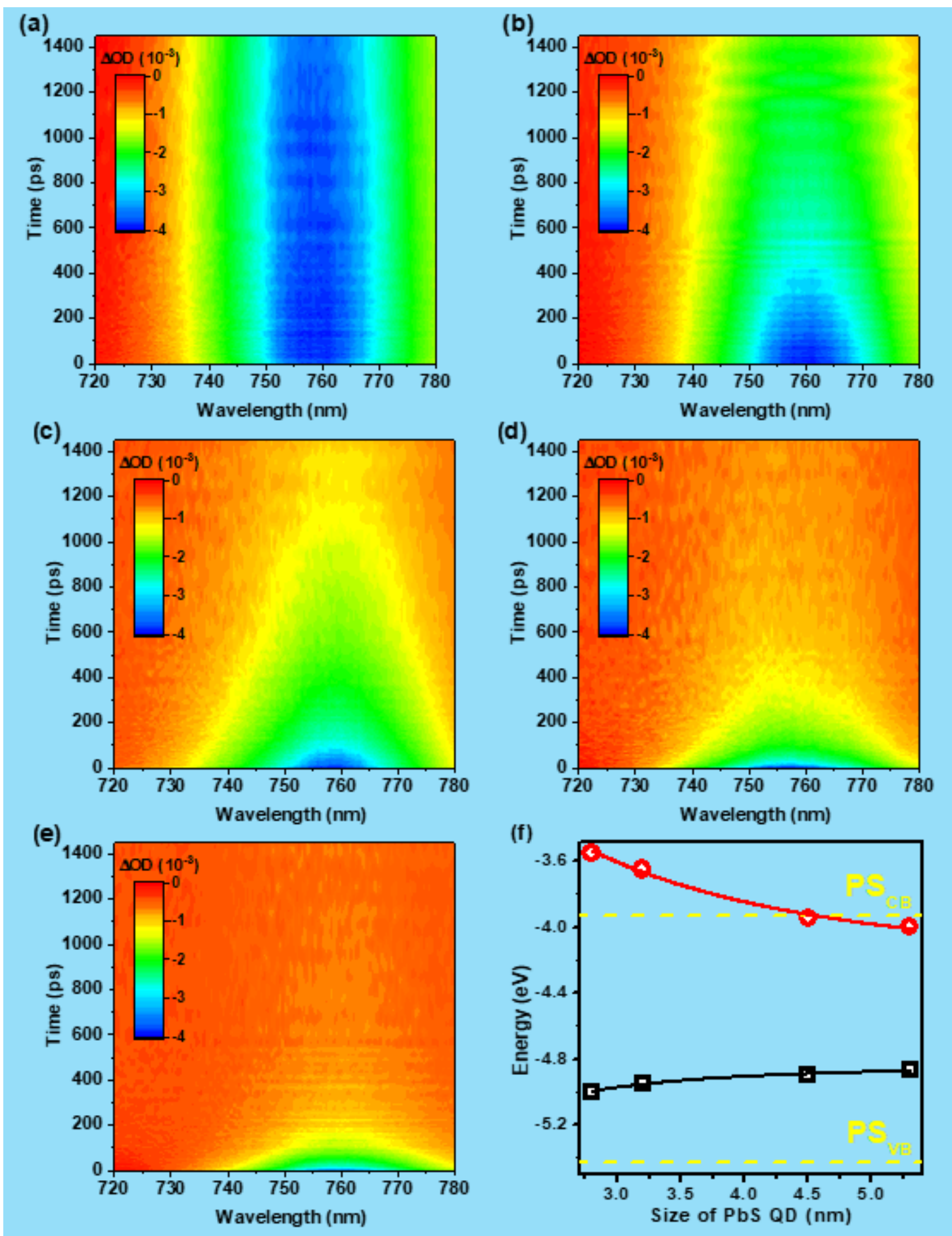

Figure 1. Two-dimensional pseudo-colour map of (a) PS, (b) PS/QDs $2.9 \mathrm{~nm}$, (c) PS/QDs 3.2 $\mathrm{nm}$, (d) PS/QDs $4.5 \mathrm{~nm}$ and (e) PS/QDs $5.3 \mathrm{~nm}$ gated at $760 \mathrm{~nm}$ in PS and PS/QDs samples having QDs of different sizes. (f) Positions of VB ( $\square$ ) and CB (o) for PbS QDs of different sizes. Representative fs-A decays corresponding to panels (a)-(e) are in the supporting information. 


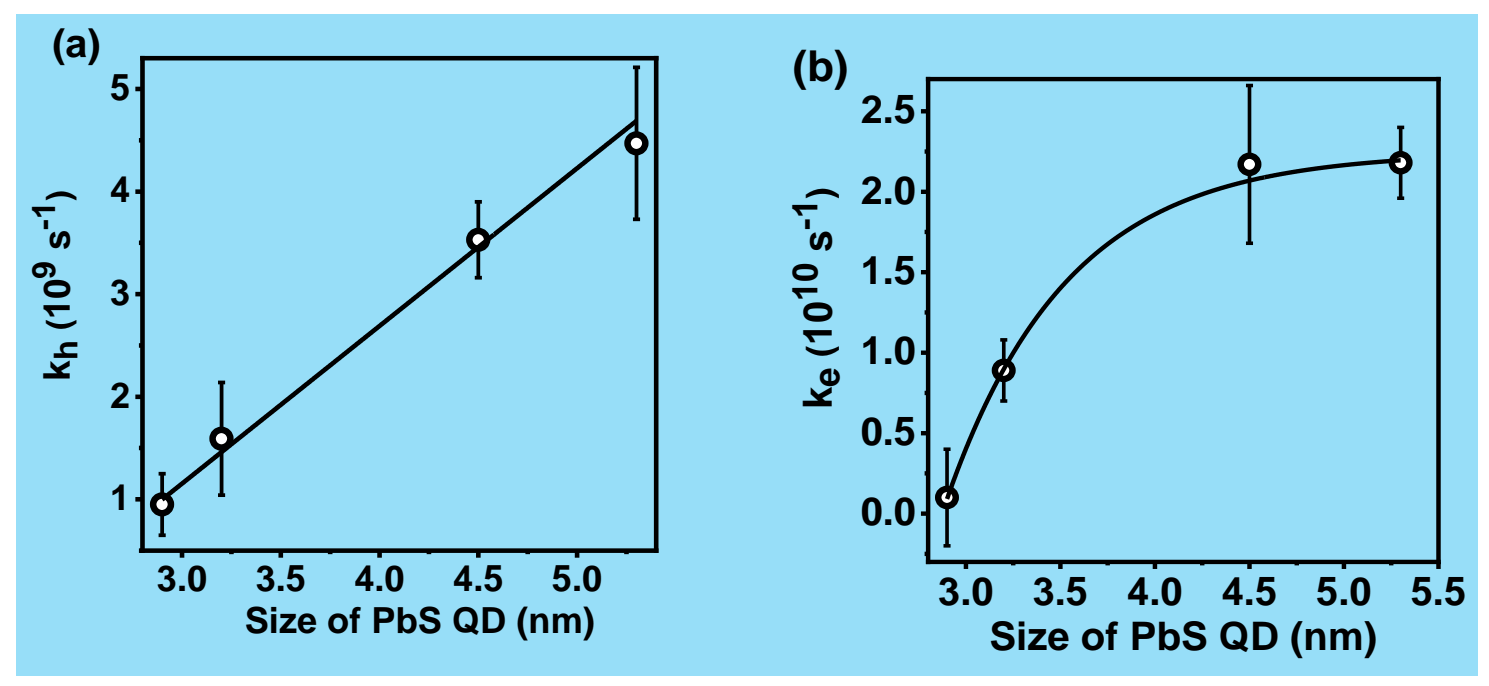

Figure 2. Experimental values of (a) holes and (b) electrons total rate constants in $\mathrm{PS} / \mathrm{PbS}$ QDs samples. The curve is to guide the eye.

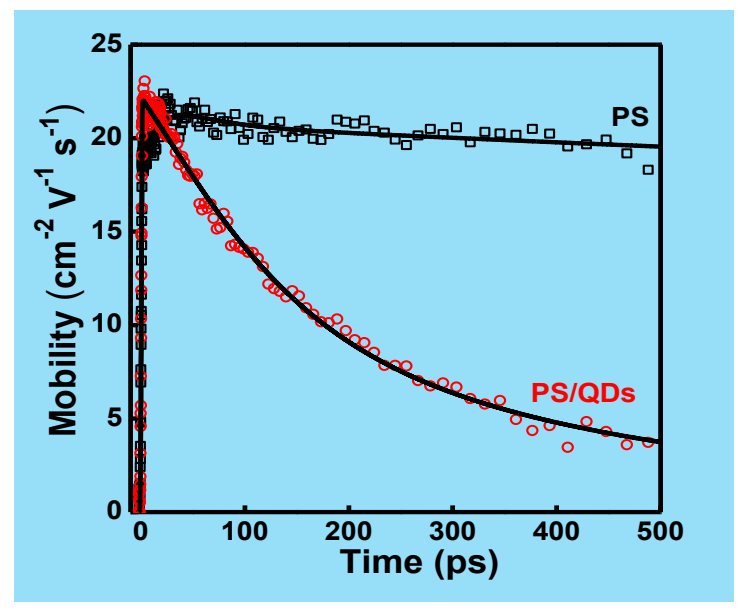

Figure 3. Femtosecond $\mathrm{THz}$ transient decays in neat PS and PS/QDs $(4.5 \mathrm{~nm})$ films. The samples were fs-excited at 600 nmwith a fluence of the absorbed photons of $8.2 \times 10^{12} \mathrm{ph} / \mathrm{cm}^{2}$. 

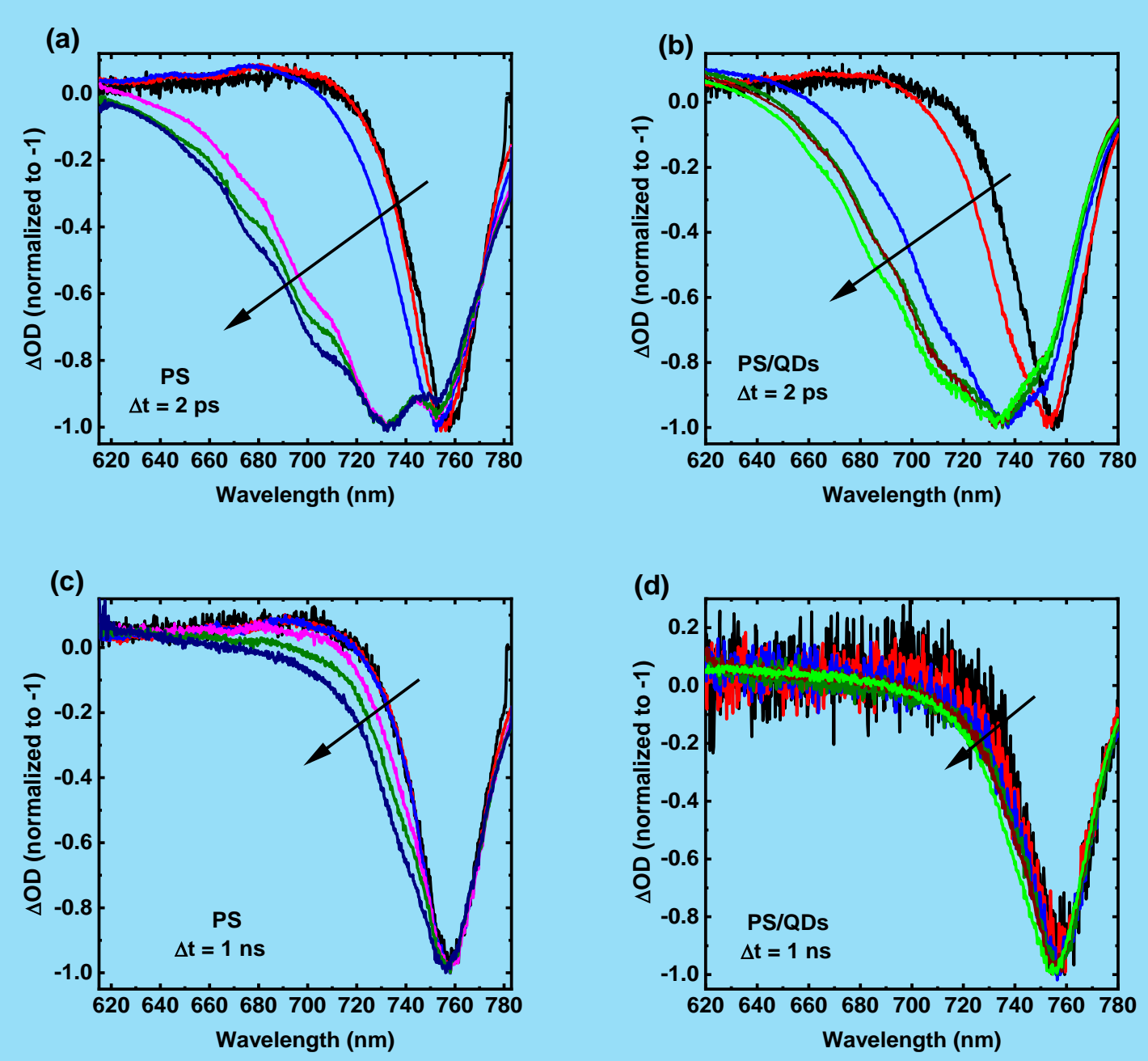

Figure 4. (a, b) TA spectra of neat PS and PS/QDs (3.2 nm) at 2 ps and (c,d) 1 ns pump-probe delay times upon excitation at $600 \mathrm{~nm}$ with fluences of absorbed photons $\left(10^{12} \mathrm{ph} / \mathrm{cm}^{2}\right): 8.2$, $41,82,410,820,1230$ for PS and 8.2, 82, 410, 820, 1030, 1230 for PS/QDs films. The arrows indicate the direction of increase of the pump fluence. 

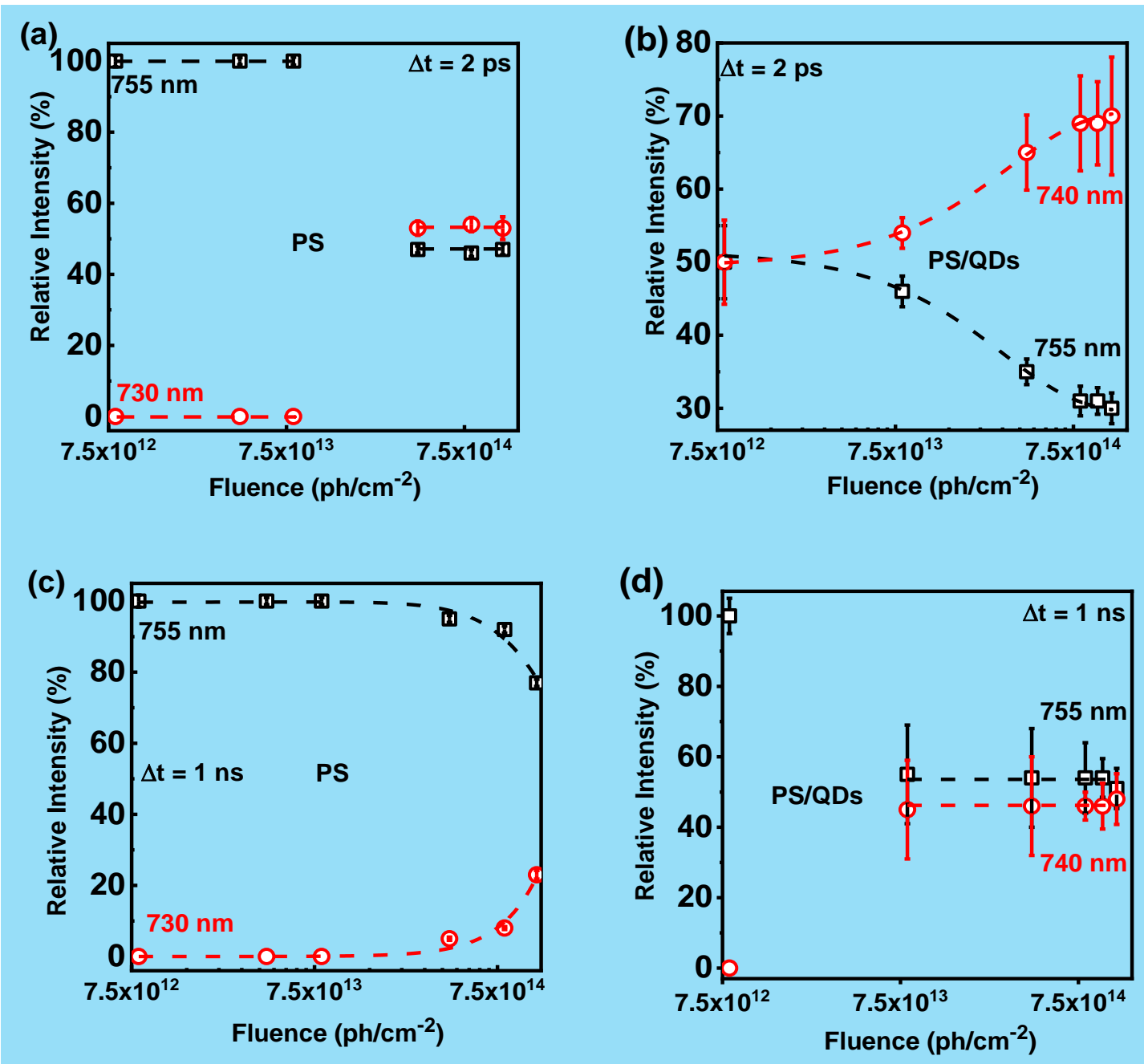

Figure 5. (a, b) Relative integral intensities of the sub-bands at 730/740 and 755 of neat PS and PS/QDs $(3.2 \mathrm{~nm})$ upon excitation at $600 \mathrm{~nm}$ with different fluences of absorbed photons at 2 ps and (c,d) 1-ns pump-probe delay-times. The sub-bands were obtained as a result of deconvolution of the bleach band using Lorentz functions. The dashed curves are for guiding the eye. 
GRAPHICAL ABSTRACT:

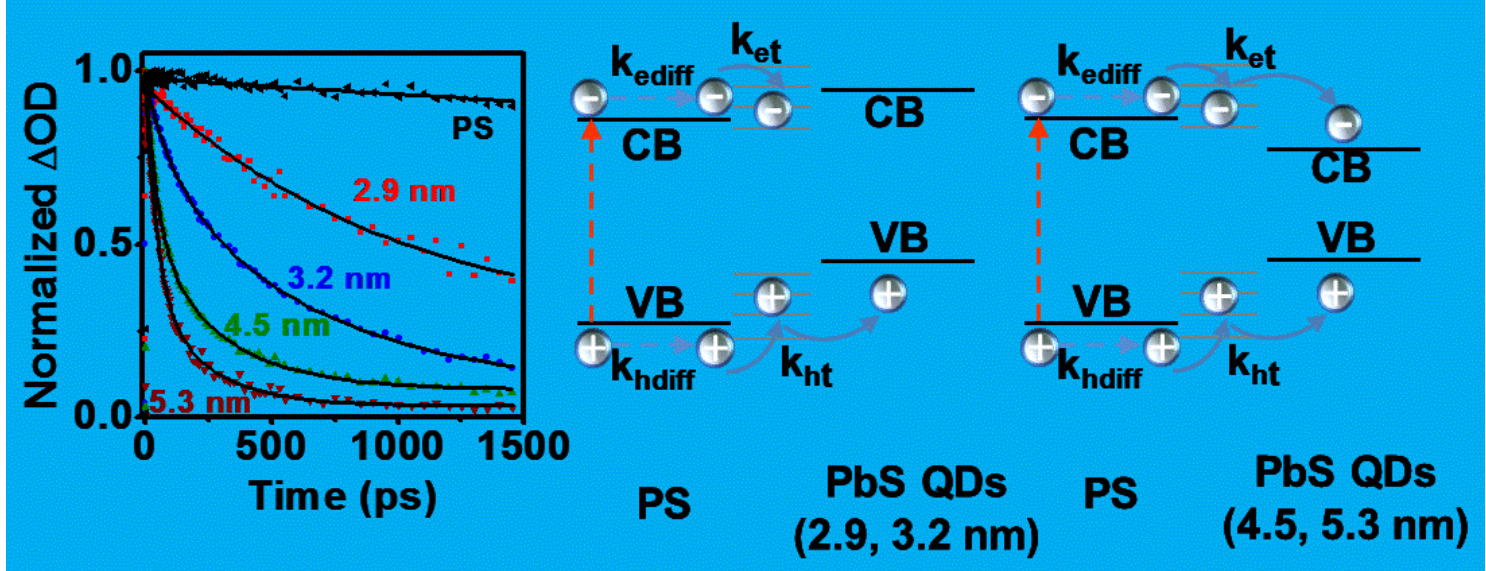

